\title{
MicroRNA-19a-3p enhances the proliferation and insulin secretion, while it inhibits the apoptosis of pancreatic $\beta$ cells via the inhibition of SOCS3
}

\author{
YUAN LI, TIANYOU LUO, LIANG WANG, JING WU and SHIXIN GUO \\ Department of Radiology, The First Affiliated Hospital of Chongqing Medical University, Chongqing 410000, P.R. China
}

Received November 11, 2015; Accepted August 22, 2016

DOI: $10.3892 /$ ijmm.2016.2748

\begin{abstract}
MicroRNAs (miRNAs or miRs), a group of small non-coding RNAs, have been demonstrated to play key roles in various physicological processes and diseases, including diabetes, the most common metabolic disorder. However, the underlying mechanisms remains largely unknown. In this study, we aimed to investigate the role of miR-19a-3p in diabetes. The results of RT-qPCR demonstrated that the level of miR-19a-3p was significantly decreased in the diabetic patients, and that the decreased miR-50a-5p level was significantly associated with a high concentration of blood glucose. miR-19a-3p mimic was further used to transfect pancreatic $\beta$ cells, and we found that the overexpression of miR-19a-3p promoted cell proliferation and insulin secretion, while it suppressed the apoptosis of pancreatic $\beta$ cells. Suppressor of cytokine signaling 3 (SOCS3) was further identified as a direct target gene of miR-19a-3p, and its protein level was significantly decreased following the overexpression of miR-19a-3p. Moreover, the siRNA-induced downregulation of SOCS3 also enhanced cell proliferation and insulin secretion, while it inhibited the apoptosis of pancreatic $\beta$ cells. In addition, the overexpression of SOCS3 reversed the effects of miR-19a-3p overexpression on cell proliferation, insulin secretion and on the apoptosis of pancreatic $\beta$ cells, which further indicates that SOCS3 acts as a downstream effector in the miR-19a-3p-mediated function of pancreatic $\beta$ cells. Finally, the level of SOCS3 was increased in diabetic patients, and inversely correlated with the miR-19a-3p level, suggesting that the downregulation of miR-19a-3p leads to the upregulation of SOCS3, which contributes to the dysfunction of pancreatic $\beta$ cells. On the whole, the findings of this study suggest that miR-19a-3p plays an important role in $\beta$ cell function, and that the miR-19a-3p/SOCS3 axis may become a potential therapeutic target for diabetes.
\end{abstract}

Correspondence to: Professor Tianyou Luo, Department of Radiology, The First Affiliated Hospital of Chongqing Medical University, 1 Youyi Road, Chongqing 410000, P.R. China

E-mail: pro.luotianyou@qq.com

Key words: diabetes, microRNA-19a-3p, proliferation, pancreatic $\beta$ cells, insulin secretion

\section{Introduction}

Diabetes mellitus, which is a complex metabolic disease, is characterized by increased blood glucose levels, which are caused by the lack of insulin production or resistance to insulin (1). The most common forms are type 1 diabetes (T1D) and type 2 diabetes (T2D) $(1,2)$. T1D is mainly caused by autoimmune $\beta$ cell destruction-induced insulin deficiency, while T2D often results from defects in insulin sensitivity and $\beta$ cell dysfunction $(1,3,4)$. However, the molecular mechanisms through which $\beta$ cell dysfunction occurs have not yet been fully elucidated. Thus, the understanding of the underlying mechanisms may aid in the development of novel therapeutic strategies for diabetes.

MicroRNAs (miRNAs or miRs), a class of non-coding RNAs, 18-25 nucleotides in length, are able to suppress gene expression by targeting the complementary regions of mRNAs and inhibiting protein translation (5). By negatively mediating their target genes, miRs act as key regulators in a variety of physiological, pathological and biological processes, including development, metabolic disorders and tumorigenesis, as well in diseases, such as diabetes (6-9). Deregulations of miRs have been observed in patients with diabetes, and specific miRs have been demonstrated to be involved in the regulation of pancreatic development and function $(6,10,11)$. For instance, Jacovetti et al found that $\beta$ cell maturation was associated with alterations in the expression of miRs induced by the nutritional transition that occurs at weaning, and suggested that miRs play a central role in post-natal $\beta$ cell maturation and in the determination of adult functional $\beta$ cell mass (12). The serum levels of miR-15a have been shown to be reduced in patients with T2D and in individuals with impaired fasting glucose (IFG)/impaired glucose tolerance (IGT), and a lower miR-15a expression has been shown to be significantly associated with T2D and pre-diabetes (13). However, the miR-mediated effectors or signaling pathways that play key roles in diabetes have not yet been fully investigated.

miR-19a-3p, a member of the miR-17-92 miR cluster, has been previously found to be a regulator of the expression of 5-lipoxygenase, a key enzyme in leukotriene biosynthesis (14). Moreover, it has been previoulsy suggested to be associated with several types of human cancer, such as breast cancer (15), astrocytoma (16), gastric cancer (17), skin cancer (18) and colorectal adenocarcinoma (19). Recently, miR-19a-3p was found to be upregulated in patients with gestational diabetes mellitus (20). 
However, its levels have been shown to be downregulated in the livers of $\mathrm{db} / \mathrm{db}$ mice, and miR-19a-3p has been suggested to promote glycogenesis in hepatocytes through the downregulation of phosphatase and tensin homolog (PTEN) expression (21). Furthermore, miR-19a has been shown to promote cell proliferation and angiogenesis by regulating the PI3K/AKT pathway associated with diabetes-associated pancreatic cancer (22-24). However, the detailed role of miR-19a-3p in the regulation of $\beta$ cell proliferation and function, as well as the underlying mechanisms, remain largely unknown.

In this study, we aimed to examine the role of miR-19a-3p in diabetes. We found that miR-19a-3p was significantly downregulated in diabetic patients, and that it enhanced cell proliferation and insulin secretion, while it inhibited the apoptosis of pancreatic $\beta$ cells by directly targeting suppressor of cytokine signaling 3 (SOCS3). Accordingly, we suggest that miR-19a-3p may serve as a potential candidate for the clinical management of diabetes.

\section{Materials and methods}

Blood sample collection. This study was approved by the Ethics Committee of the First Affiliated Hospital of Chongqing Medical University (Chongqing, China), and was carried out in accordance with the Declaration of Helsinki. Written informed consent was obtained from all study subjects prior to enrollment. Blood samples from patients with T2D $(n=45)$ and normal subjects $(n=20)$ were collected from the First Affiliated Hospital of Chongqing Medical University from November, 2012 to February, 2014. Patients with serious liver or kidney diseases, malignancy and acute heart failure were excluded.

Determination of glucose and SOCS3 levels in blood samples. The glucose levels in the blood samples were determined by the routine laboratory method. The blood glucose levels were examined at the Department of Clinical Laboratory of our hospital using the Glucose Assay kit (BioVision, San Francisco, CA, USA), according to the manufacturer's instructions. Plasma SOCS3 levels were analyzed by enzyme-linked immunosorbent assay (ELISA), according to the manufacturer's instructions using the SOCS-3 ELISA kit (Ybiotech, Shanghai, China). Briefly, an equal amount of protein $(50 \mu \mathrm{g})$ was diluted with sample dilution buffer to $100 \mu \mathrm{l}$, and then added in each well, which was incubated for $2.5 \mathrm{~h}$ at room temperature with gentle shaking. The solution was discarded and washed for 4 times with wash solution. After the final wash, any remaining liquid was removed by decanting. The plate was then inverted and blotted against clean paper towels. SOCS3 detection antibody was then added followed by incubation for $1 \mathrm{~h}$ at room temperature with gentle shaking. Subsequently, $100 \mu \mathrm{l}$ of HRP-streptavidin solution was added followed by incubation for $45 \mathrm{~min}$ at room temperature with gentle shaking. This was followed by another wash and $100 \mu \mathrm{l}$ of TMB one-Step substrate reagent was added to each well followed by incubation for $30 \mathrm{~min}$ at room temperature in the dark with gentle shaking. Subsequently, $50 \mu 1$ of stop solution was added and read at $450 \mathrm{~nm}$ immediately. A standard curve was constructed using various dilutions of the SOCS3 standard preparation in the kit. The content of SOCS3 was determined by extrapolation of the absorbance to the standard curve.
Cell culture. The pancreatic $\beta$ cell lines, INS-1 and MIN6, were purchased from the Cell Bank of the Chinese Academy of Sciences (Shanghai, China). The cells were cultured in Dulbecco's modified Eagle's medium (DMEM) supplemented with $25 \mathrm{mM}$ glucose and $15 \%$ fetal bovine serum (FBS) (both from Life Technologies, Grand Island, NY, USA) and $5.5 \mathrm{mM}$ 2-mercaptoethanol (Thermo Fisher Scientific, Waltham, MA, USA). INS-1 and MIN6 cells were collected and then suspended using DMEM with 10\% FBS. The cells were then seed into a 6-well plate (300,000 cells per well), and cultured to $70 \%-80 \%$ confluence.

Cell transfection. miR-negative control (miR-NC), miR-19a-3p mimic (both from GenePharma, Shanghai, China), siRNA targeting SOCS3 (SOCS3 siRNA), non-specific siRNA (NC siRNA), the pc-DNA3.1-SOCS3 plasmid and pc-DNA3.1 vector (NC), and Lipofectamine 2000 were diluted with OPTI-MEM (both from Life Technologies). The diluted Lipofectamine 2000 was added to the respective diluted plasmid, $\mathrm{miR}$, or siRNA. Following incubation at room temperature for $20 \mathrm{~min}$, the above mixture was added to the cell suspension, which was then incubated at $37^{\circ} \mathrm{C}, 5 \% \mathrm{CO}_{2}$ for $6 \mathrm{~h}$. Subsequently, the transfection mixture was replaced by DMEM with $10 \%$ FBS. Untransfected cells were used as controls. Following transfection for $48 \mathrm{~h}$, the following assays were conducted:

RNA isolation and reverse transcription-quantitative polymerase chain reaction $(R T-q P C R)$. Total RNA was extracted from the cells using TRIzol reagent (Life Technologies). RT-qPCR was used to examine the relative miR-19a-3p expression using the mirVana ${ }^{\mathrm{TM}}$ real-time RT-PCR microRNA detection kit (Life Technologies), in accordance with the manufacturer's instructions. U6 was used as an internal reference. The specific primers for miR-19a-3p and U6 were purchased from Genecopoeia, Guangzhou, China. The relative mRNA expression of SOCS3 was detected by quantitative PCR (qPCR) using the standard SYBR-Green RT-PCR kit (Takara, Otsu, Japan) in accordance with the manufacturer's instructions. Glyceraldehyde 3-phosphate dehydrogenase (GAPDH) was used as an internal reference gene. The specific primers for SOCS3 were as follows: forward, 5'-ATGGTCACCCACAGCAAGTTT-3' and reverse, 5'-TCC AGTAGAATCCGCTCTCCT-3'. The specific primers for GAPDH were as follows: forward, 5'-TGGCCTTCCGT GTTCCTAC-3' and reverse, 5'-GAGTTGCTGTTGAAGT CGCA-3'. The relative expression level was quantified using the the $2^{-\Delta \Delta \mathrm{Ct}}$ method.

Western blot analysis. The cells were lysed in the protein lysis buffer [50 mM Tris/HCl, pH 8.0, $250 \mathrm{mM} \mathrm{NaCl}, 1 \% \mathrm{NP}-40$, $0.5 \%$ (w/v) sodium deoxycholate, $0.1 \%$ sodium dodecylsulfate, $1 \%$ PMSF and $1 \mathrm{X}$ phosphatase inhibitor cocktail]. The protein concentration was determined using the BCA Protein assay kit (Pierce Chemical, Rockford, IL, USA). Protein $(60 \mu \mathrm{g})$ was separated by $10 \%$ sodium dodecyl sulfate-polyacrylamide gel electrophoresis (SDS-PAGE), transferred onto a PVDF membrane (Life Technologies), and then blocked in 5\% non-fat dried milk (Yili, Beijing, China) in TBST for $2 \mathrm{~h}$. The PVDF membrane was then incubated with primary antibodies against SOCS3 (ab16030) and GAPDH (ab37168; both from Abcam, 


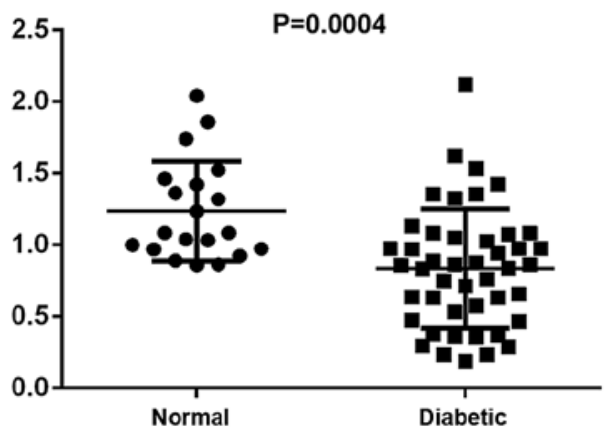

B

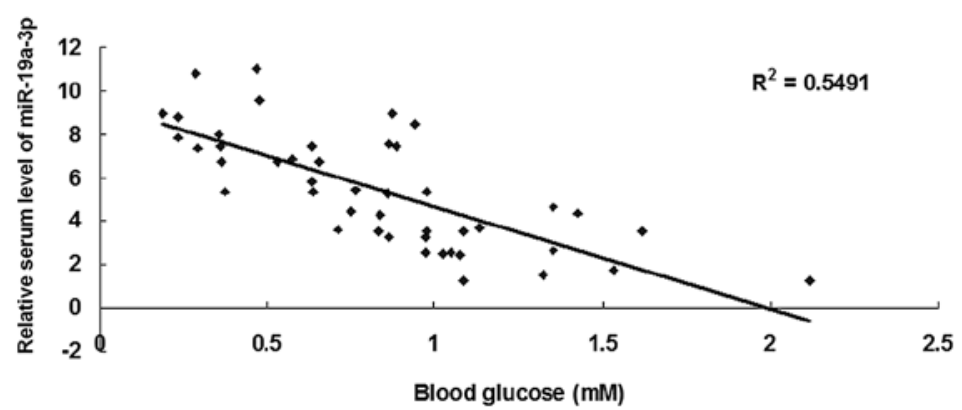

Figure 1. (A) RT-qPCR was used to detect the miR-19a-3p level in the blood samples from type 2 diabetes patients (n=45) and normal subjects (n=20). (B) A significant inverse correlation was observed between the plasma miR-19a-3p level and the blood glucose concentration among patients with type 2 diabetes ( $\mathrm{n}=45$ ).

Cambridge, MA, USA) at $4^{\circ} \mathrm{C}$ overnight, and then washed with TBST 4 times. The PVDF membrane was then incubated with the corresponding secondary antibody (goat monoclonal anti-rabbit IgG; ab190492; Abcam) for $1 \mathrm{~h}$ at room temperature, and then washed with TBST 3 times. The immune complexes were then detected using the ECL Western Blotting kit (Pierce Chemical) and X-film (Kodak, Tokyo, Japan). ImageJ software was used to analyze the relative protein expression, represented as the density ratio versus GAPDH.

Detection of glucose-stimulated insulin secretion. The cells were seeded in a 96-well plate and cultured overnight. The cells were then treated with basal glucose $(3.3 \mathrm{mmol} / \mathrm{l})$ or stimulatory glucose $(16.7 \mathrm{mmol} / \mathrm{l})$ for $1 \mathrm{~h}$. Subsequently, the insulin level was measured by ELISA. In brief, the cells in each well were sonicated in acid ethanol, followed by 3 freeze/thaw cycles, and then centrifuged for $5 \mathrm{~min}$ at $10,000 \mathrm{x} \mathrm{g}$. The supernatant was used to measure the insulin level by ELISA as described above.

Determination of cell proliferation. The cells $\left(2 \times 10^{3}\right)$ in each group (miR-NC, miR-19a-3p mimic, SOCS3 siRNA, NC siRNA, miR-19a-3p mimic + SOCS3, ormiR-19a-3p mimic + NC vector) were plated into a 96-well plate and cultured for 1 to 5 days at $37^{\circ} \mathrm{C}$ with $5 \% \mathrm{CO}_{2}$. Subsequently, $20 \mu \mathrm{l}$ of 3-(4,5-dimethylthiazol-2-yl)-2,5-diphenyltetrazolium bromide (MTT; $5 \mathrm{mg} / \mathrm{ml}$; Sigma, St. Louis, MO, USA) were added. Followoing incubation at $37^{\circ} \mathrm{C}$ for $4 \mathrm{~h}, 150 \mu \mathrm{l}$ of dimethyl sulfoxide (DMSO) were added. Following incubation at room temperature for $10 \mathrm{~min}$, the formazan production was detected by determining the optical density (OD) at $570 \mathrm{~nm}$ using the Elx800 enzyme immunoassay analyzer (BioTek Instruments, Inc., Winooski, VT, USA).

Apoptosis assay. The cell apoptotic levels were examined using the Annexin V-fluorescein isothiocyanate (FITC) apoptosis detection kit (BD Pharmingen, San Diego, CA, USA), according to the manufacturer's instructions. The cells in each group were re-suspended in 1X binding buffer solution with Annexin V-FITC and PI and incubated for $15 \mathrm{~min}$ at room temperature in the dark. The apoptotic rate was determined using the EPICS Altra flow cytometer (Beckman Coulter, Brea, CA, USA).

Bioinformatics analysis. Bioinformatics analysis was conducted to predict the putative targets of miR-19a-3p using TargetScan 4.2 online software (www.targetscan.org).
Luciferase reporter assay. The wild-type (WT) of SOCS3 3'-UTR was constructed by PCR and inserted into the pMIR-REPORT miRNA Expression Reporter vector (Ambion, Carlsbad, CA, USA). The mutant type (MT) of SOCS3 3'-UTR was constructed using the Easy Mutagenesis system kit (Promega, Madison, WI, USA), in accordance with the manufacture's instructions, and inserted into the pMIR-REPORT miRNA Expression Reporter vector. 293 cells (Cell Bank of the Chinese Academy of Sciences) were plated in 96-well plates, and co-transfected with the WT SOCS3-3'UTR or MT SOCS3-3'UTR plasmid (300 ng), and miR-NC or miR-19a-3p mimic (100 nM), using Lipofectamine 2000, in accordance with the manufacture's instructions. The dualluciferase reporter assay system (Promega) was used to determine the activity of Renilla luciferase and Firefly luciferase following co-transfection for $48 \mathrm{~h}$. The Renilla luciferase activity was normalized to the Firefly luciferase activity.

Statistical analysis. The data of at least 3 independent experiments are expressed as the means \pm SD. GraphPad Prism 5 software (GraphPad Software, Inc., La Jolla, CA, USA) was used to perform statistical analysis. The differences were analyzed using the Student's t-test or one-way ANOVA. The association between the miR-19a-3p level and the blood glucose or SOCS3 levels was analyzed using Spearman's correlation coefficient. A value of $\mathrm{P}<0.05$ was considered to indicate a statistically significant difference.

\section{Results}

miR-19a-3p expression is significantly downregulated in diabetic patients. To reveal the function of miR-19a-3p in diabetes, we first conducted RT-qPCR to examine the plasma miR-19a-3p level in diabetic patients and normal subjects. Our data demonstrated that the miR-19a-3p level was significantly decreased in the blood of diabetic patients, when compared with that of normal subjects (Fig. 1A). In addition, we observed a significant inverse correlation between the plasma miR-19a-3p level and the blood glucose concentration among these diabetic patients (Fig. 1B). These data suggest that the downregulation of miR-19a-3p expression plays a role in the progression of diabetes.

miR-19a-3p enhances the proliferation and insulin secretion, while it inhibits the apoptosis of pancreatic $\beta$ cells. The 

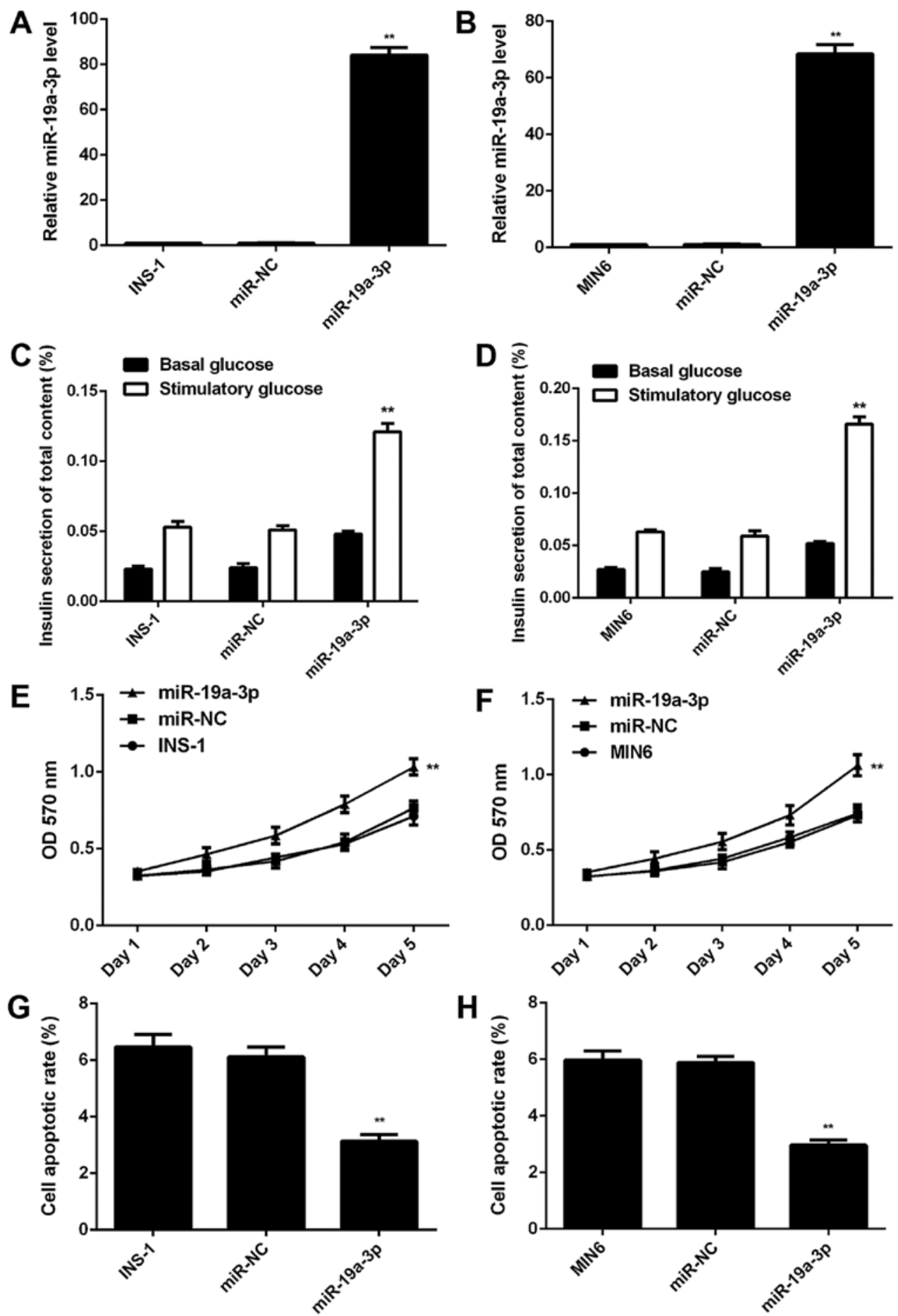

Figure 2. RT-qPCR was used to detect the miR-19a-3p level in (A) INS-1 and (B) MIN6 cells transfected with scramble miR mimic (miR-NC) or miR-19a-3p mimic. Untransfected INS-1 or MIN6 cells were used as a control. (C and D) Glucose-stimulated insulin secretion was then determined in each group. (E and F) MTT assay and (G and H) flow cytometry were then performed to examine cell proliferation and apoptosis. ${ }^{* *} \mathrm{P}<0.01$ vs. INS-1 or MIN6 untransfected cells (bars labeled as INS-1 or MIN6) or negative control (NC)-transfected cells.

glucose-responsive pancreatic $\beta$ cell lines, INS-1 and MIN6, were used in our in vitro experiments to investigate the exact role of miR-19a-3p in pancreatic $\beta$ cells. As miR-19a-3p was found to be significantly downregulated in diabetic patients, we transfected the INS-1 and MIN6 cells with miR-19a-3p mimic to restore its expression level. Following transfection, qPCR was conducted to examine the expression level of miR-19a-3p. Our data indicated that the level of miR-19a-3p was significantly higher in the cells transfected with miR-19a-3p mimic compared to the control or negative control (NC) groups (Fig. 2A and B). However, transfection with miR-NC did not alter the miR-19a-3p level in the INS-1 and MIN6 cells (Fig. 2A and B). We then found that the insulin secretion in response to glucose stimulation was significantly increased in the miR-19a-3p-overexpressing INS-1 and MIN6 cells, when compared with the control and NC groups (Fig. 2C and D), indicating that miR-19a-3p induced insulin secretion under conditions of glucose stimulation. We 
A
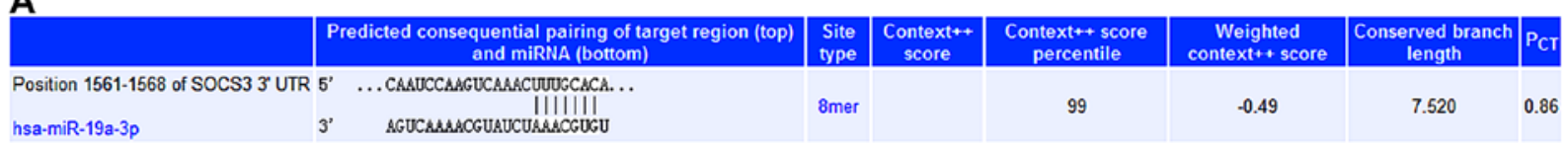

B $\ldots \ldots 1530 \ldots \ldots \ldots 1540 \ldots \ldots \ldots \ldots 1550 \ldots \ldots 1560 \ldots \ldots \ldots 1570 .$.

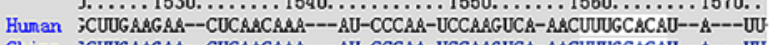
Chimp ;CUUGRAGAS--CUCAACARA---AU-CCCAR-UCCAAGUCA-AACUUUGCACAU--A---UU Rhesus ;CUUGAAGAR--CUCAACAAA---AU-CCCAA-UCCAAGUCA-AACUUUGCACAU--A---UU

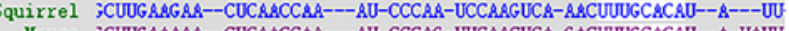

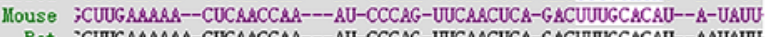
Rat ;CUUGARARAS-CUCAACCAR---AU-CCCAG-UUCAACUCA-GACUUUGCACAU-AAUAUU

Rabbit ;CUUGRAGAS-CUCARCCAR---AU-CCOGA-CCCCAGUCA-ARCUUUGCACAU-- $---U U$

Pig ;CUUGARAAS-CUCAACGAA--AU-ACCAA-UCCAAGUCA-AACUUUGCACAU-D---UU

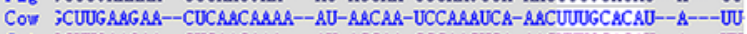

Cat ;CUUGARGAS-CUCARCARA---AU-ACCAR-CCCARCUCA-ABCUUUGCACAD-- - ---UU

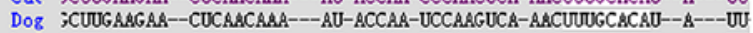

Brown bat ;CUUGAS----CUCAACCAA---AU-ACCAA-UCCAAGUCA-AACUUUGCACAU-- - ---UU

Elephant ;CUUGAAGAS-CUCAACAAA---AU-ACCAA-UUCAAGUCA-AACUUUGCACAU--

Opossun ;CUUGAAGA--COCAACAGA---AU-ACAAA-UUGAAGUCA-AACUUUGCACAD-- $\mathbf{B}^{--}-\mathrm{UU}$

Macav

Chicken ;CUUGAA-A---UUCAACCAA---AA-CAAAA-CUCAGAUGA-AACUUUGCACAU--A---UU

Lizard ;CUUGAR-GA--CUCAACCAA---ARAUCAAA-CUUGGAAGAAAACUUUGCACAUCUA---UU

X. tropicalis ---CAAC---AU-C----CCCAGGACA-AAAUUUGCACAU-- $----U$ Con jCUUGARGR. . CUCARCARA. . . AU. CCCAR. UCCARGUCA. ARCUUUGCACAU. . A. . . UU

C

WT SOCS3 3'-UTR 5'...UCAAACUUUGCACA...3' hsa-miR-19a-3p 3'...GUAUCUAAACGUGU...5 |||| $\mid$

MT SOCS3 3'-UTR 5'...UCAAACCCCGCACA...3'

\section{E}
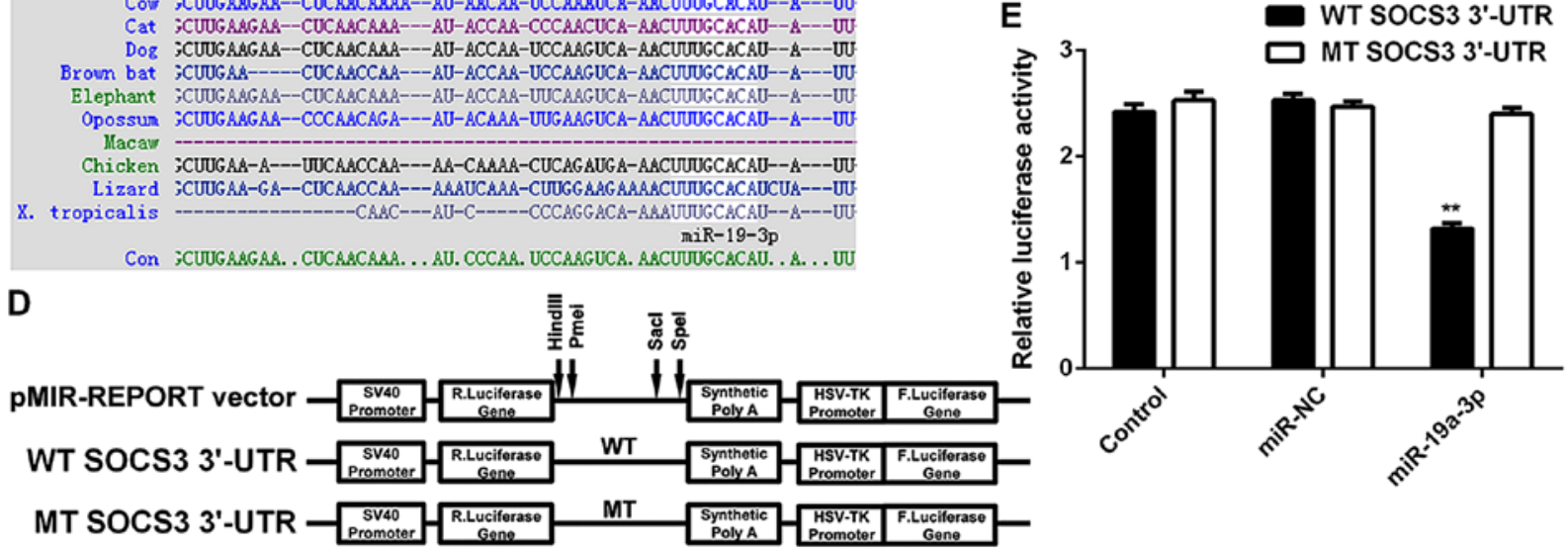

$\mathbf{F}$
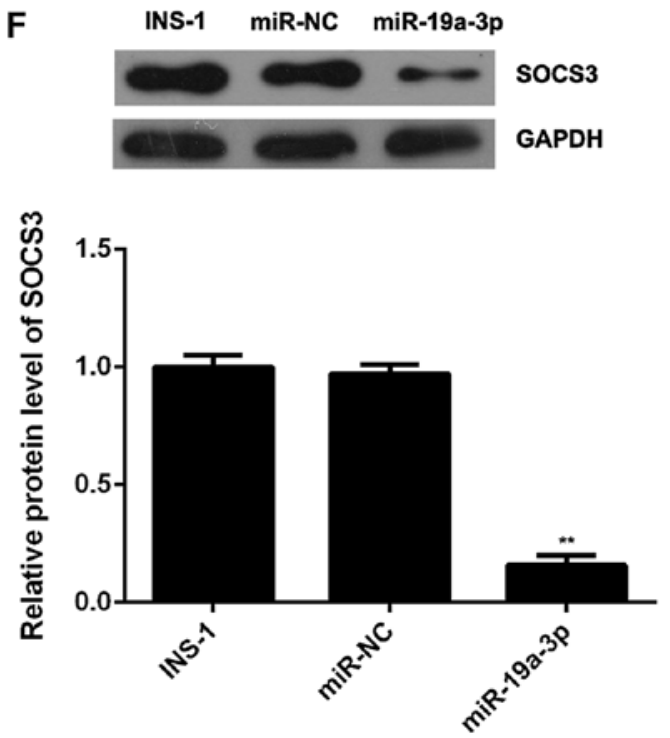

G
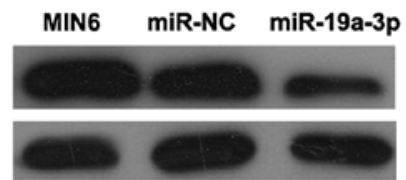

socs 3

Figure 3. (A and B) SOCS3 was predicated to be a potential target gene of miR-19a-3p with evolutionary conservation. (C and D) The wild-type (WT) SOCS3 3'-UTR containing the putative miR-19a-3p binding sites or the mutant type (MT) SOCS3 3'-UTR without the predicated miR-19a-3p binding sites were subcloned into the pMiR-REPORT miRNA Expression Reporter vector. (E) Luciferase reporter data indicated that co-transfection with the WT SOCS3 3'-UTR vector and miR-19a-3p mimic caused a significant decrease in luciferase activity; however, the luciferase activity was not altered in the 293 cells co-transfected with the MT SOCS3 3'-UTR vector and miR-19a-3p mimic. ${ }^{* *} \mathrm{P}<0.01$ vs. controls (untransfected cells or miR-NC-transfected cells). (F and G) The protein expression of SOCS3 was examined by western blot analysis in INS-1 and MIN6 cells transfected with negative control miR mimic (miR-NC) or miR-19a-3p mimic. ${ }^{* *} \mathrm{P}<0.01$ vs. INS-1 or MIN6 untransfected cells (bars labeled as INS-1 or MIN6) or negative control (NC)-transfected cells.

further examined the effect of miR-19a-3p on the proliferation and apoptosis of pancreatic $\beta$ cells. Our data indicated that the overexpression of miR-19a-3p markedly enhanced cell proliferation (Fig. 2E and F), while it suppressed the apoptosis of the INS-1 and MIN6 cells (Fig. 2G and H), when compared with the control and NC groups. Based on these data, it is thus evident that miR-19a-3p enhances the proliferation and insulin secretion, while it inhibits the apoptosis of pancreatic $\beta$ cells.
SOCS3 is a target gene of miR-19a-3p in pancreatic $\beta$ cells. As miRs function by regulating the expression of their target genes, we then used TargetScan software to analyze the putative target genes of miR-19a-3p. SOCS3 was predicated to be a potential target gene of miR-19a-3p with evolutionary conservation (Fig. 3A and B). To verify their targeting relationship, the WT SOCS3 3'-UTR containing the predicated miR-19a-3p binding sites or the MT SOCS3 3'-UTR without 
the predicated miR-19a-3p binding sites were subcloned into the luciferase reporter vector, respectively (Fig. 3C and D). Luciferase reporter assay was then conducted, and our data indicated that co-transfection with the WT SOCS3 3'-UTR vector and miR-19a-3p mimic led to a significant decrease in luciferase activity; however, the luciferase activity was not altered in the 293 cells co-transfected with the MT SOCS3 3'-UTR vector and miR-19a-3p mimic (Fig. 3E). Accordingly, SOCS3 is a direct target gene of miR-19a-3p. As miRs negatively mediate the expression of their target genes at the post-transcriptional level, we then examined the protein expression of SOCS3 in miR-19a-3p-overexpressing INS-1 and MIN6 cells. We observed that the protein level of SOCS3 was markedly decreased following the upregulation of miR-19a-3p, when compared with the control and NC groups (Fig. 3F and G). Accordingly, the overexpression of $\mathrm{miR}-19 \mathrm{a}-3 \mathrm{p}$ inhibited the protein expression of SOCS3 in pancreatic $\beta$ cells.

SOCS3 is involved in the miR-19a-3p-mediated proliferation and apoptosis of, as well as in insulin secretion by pancreatic $\beta$ cells. As the overexpression of miR-19a-3p led to a significant decrease in the protein level of SOCS3, we investigated whether the downregulation of SOCS3 has similar effects as miR-19a-3p overexpression in pancreatic $\beta$ cells SOCS3 siRNA was used to transfect the INS-1 and MIN6 cells, and the protein level of SOCS3 was found to be significantly decreased following transfection (Fig. 4A and B). We further found that the knockdown of SOCS3 led to a significant increase in glucose-stimulated insulin secretion and cell proliferation, as well as to a marked decrease in the apoptosis of the INS-1 and MIN6 cells (Fig. 4C-H); these effects were very similar to those observed with miR-19a-3p overexpression. According to these data, we hypothesized that SOCS3 may act as a downstream effector of SOCS3 in pancreatic $\beta$ cells. To verify this hypothesis, the pcDNA3.1-SOCS3 plasmid was used to transfect miR-19a-3p-overexpressing INS-1 and MIN6 cells in order to restore the expression of SOCS3. Following transfection, western blot analysis was used to examine the protein level of SOCS3. As shown in Fig. 5A and B, the SOCS3 protein level was significantly higher in the INS-1 and MIN6 cells co-transfected with the miR-19a-3p mimic and SOCS3 plasmid, when compared to the cells transfected with miR-19a-3p and the NC vector. We then examined the glucose-stimulated insulin secretion, cell proliferation and cell apoptosis in each group. Our data revealed that compared with the cells transfected with miR-19a-3p and the NC vector, the INS-1 and MIN6 cells co-transfected with the miR-19a-3p mimic and SOCS3 plasmid exhibited a decrease in glucosestimulated insulin secretion and cell proliferation, and an increase in apoptosis (Fig. 5C-H). Taken together, these data demonstrate that miR-19a-3p enhances the proliferation and insulin secretion, while it inhibits the apoptosis of pancreatic $\beta$ cells by directly targeting SOCS3.

SOCS3 is upregulated in diabetic patients and its plasma level inversely correlates with miR-19a-3p. Finally, we examined the plasma SOCS3 level using ELISA. We found that the SOCS3 level was significantly increased in the blood samples of diabetic patients compared with those of normal subjects (Fig. 6A). After that, we analyzed the correlation between the plasma level of SOCS3 and miR-19a-3p. Our data indicated a significant negative correlation between the protein level of SOCS3 and the miR-19a-3p level in the blood samples of diabetic patients (Fig. 6B). Therefore, we suggest that the dysregulation of the miR-19a-3p/SOCS3 axis is involved in the development of T2D.

\section{Discussion}

Diabetes poses one of the most severe threats to human health worldwide, as it can induce high incidence rates of a number of complications. In recent years, various miRs, such as miR-375, miR-7, miR-124a2, miR-195, miR-126, miR-9, miR-96 and miR-34a have been reported to regulate the expression of a variety of genes that participate in the regulation of cell proliferation and apoptosis, as well as glucose-stimulated insulin secretion, and thus may play a role in pancreatic development and diabetes (22). For instance, treatment with interleukin (IL)-1 $\beta$ has been shown to enhance the expression of miR-101a and miR-30b in pancreatic $\beta$ cells, and the two miRs have been shown to inhibit the insulin content, and to increase $\beta$ cell death (25). Recently, miR-19a was found to be downregulated in the livers of diabetes $\mathrm{db} / \mathrm{db}$ mice and mice injected with IL-6, an inducer of insulin resistance (21). However, the exact role of miR-19a in the regulation of pancreatic $\beta$ cell proliferation, apoptosis and insulin secretion has not been studied to date, at least to the best of our knowledge.

In the present study, the data from RT-qPCR demonstrated that the miR-19a-3p level was significantly decreased in the blood of diabetic patients compared with that of normal subjects. Moreover, a significant inverse correlation was observed between the plasma miR-19a-3p level and the blood glucose concentration among the diabetic patients, suggesting that the dysregulation of miR-19a-3p is associated with the progression of diabetes. INS-1 and MIN6 are glucose-responsive murine pancreatic $\beta$ cell lines. Therefore, to further reveal the role of miR-19a-3p in pancreatic $\beta$ cell function, the INS-1 and MIN6 cells were transfected with miR-19a-3p mimic. We found that the overexpression of miR-19a-3p promoted the proliferation and insulin secretion, while it suppressed the apoptosis of INS-1 and MIN6 cells, which further supports the notion that miR-19a-3p plays a role in diabetes, consistent with the clinical data.

Previous studies have mainly focused on the role of miR-19a-3p in human cancers. Yang et al demonstrated that miR-19a-3p inhibited breast cancer progression and metastasis by inducing macrophage polarization through the targeting of the oncogene, Fra-1 (15). These data indicate that miR-19a-3p participates in the regulation of cell motility. Moreover, miR-19a-3p expression has been shown to be significantly downregulated following multifractionated radiation in breast cancer cells, and that it may contribute to radiosensitivity and can be used as a biomarker for radiotherapy (26). Furthermore, miR-19a-3p has been shown to be downregulated in nonmelanoma skin cancer, suggesting a tumor suppressor role (18). On the contrary, a high serum level of miR-19a-3p was found to be significantly associated with a poor survival of astrocytoma patients, suggesting that it may play an oncogenic role in astrocytoma (16). In addition, Zheng et al identified a four-miRNA panel, including miR-19a-3p, miR-223-3p, miR-92a-3p and miR-422a with a high diagnostic accuracy for early-stage 
A

INS-1 NC SIRNA SOCS3 SiRNA

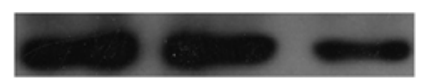

$\operatorname{socs} 3$

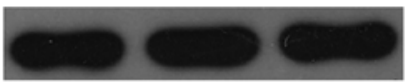

GAPDH

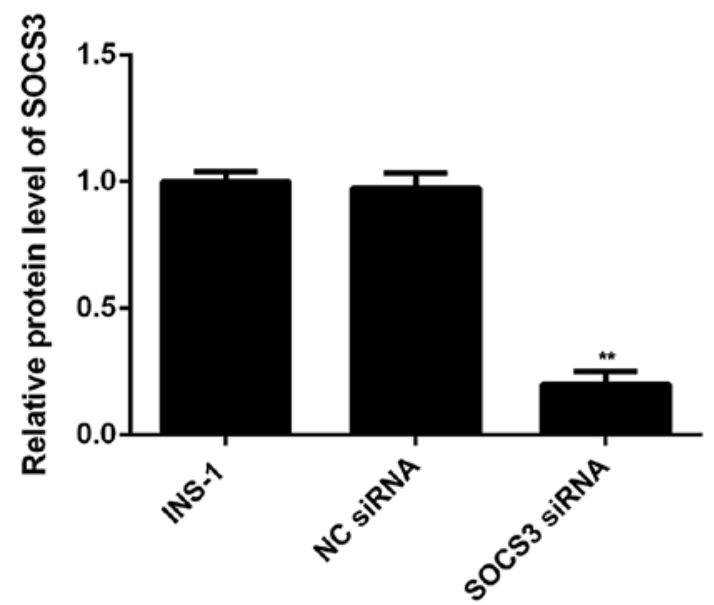

C
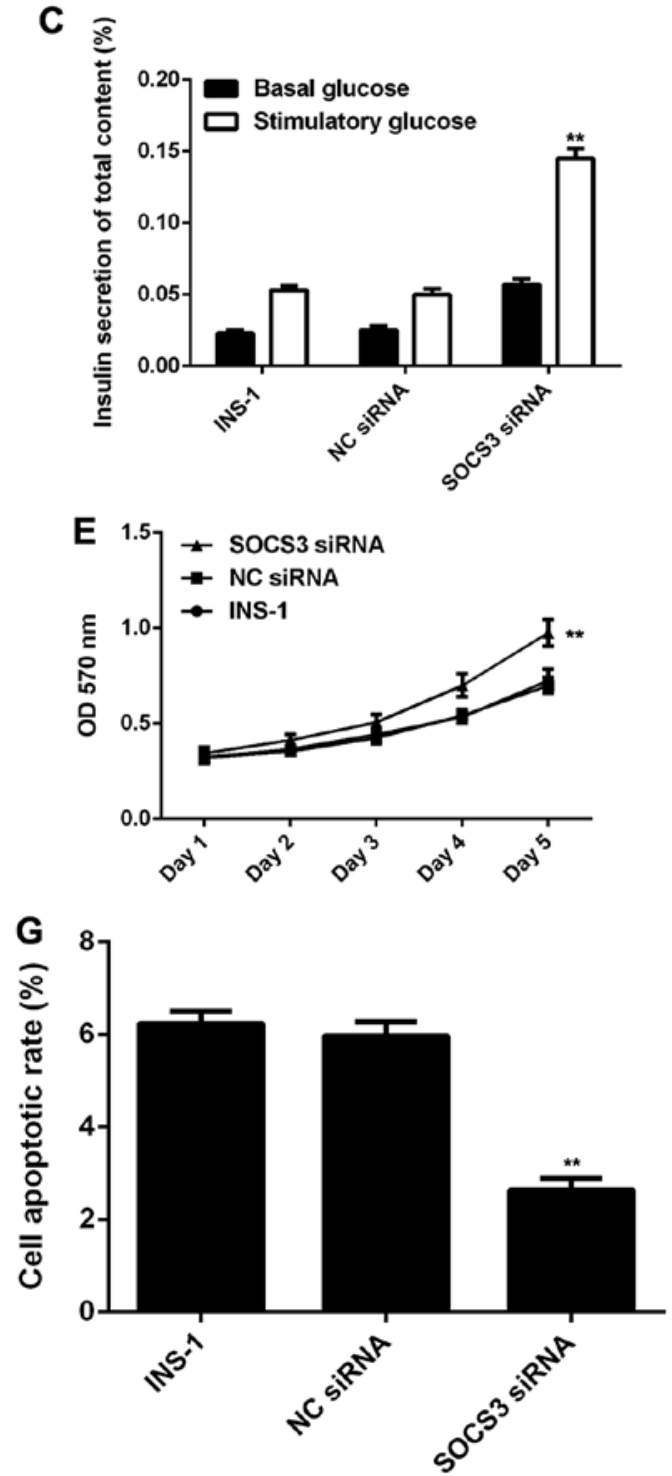

B

INS-1 NC SIRNA SOCS3 SiRNA
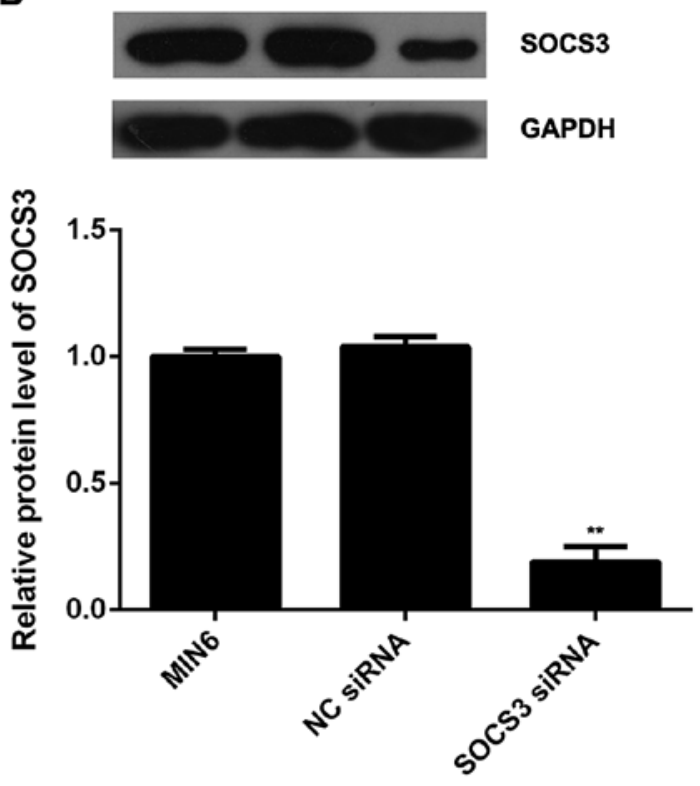

D
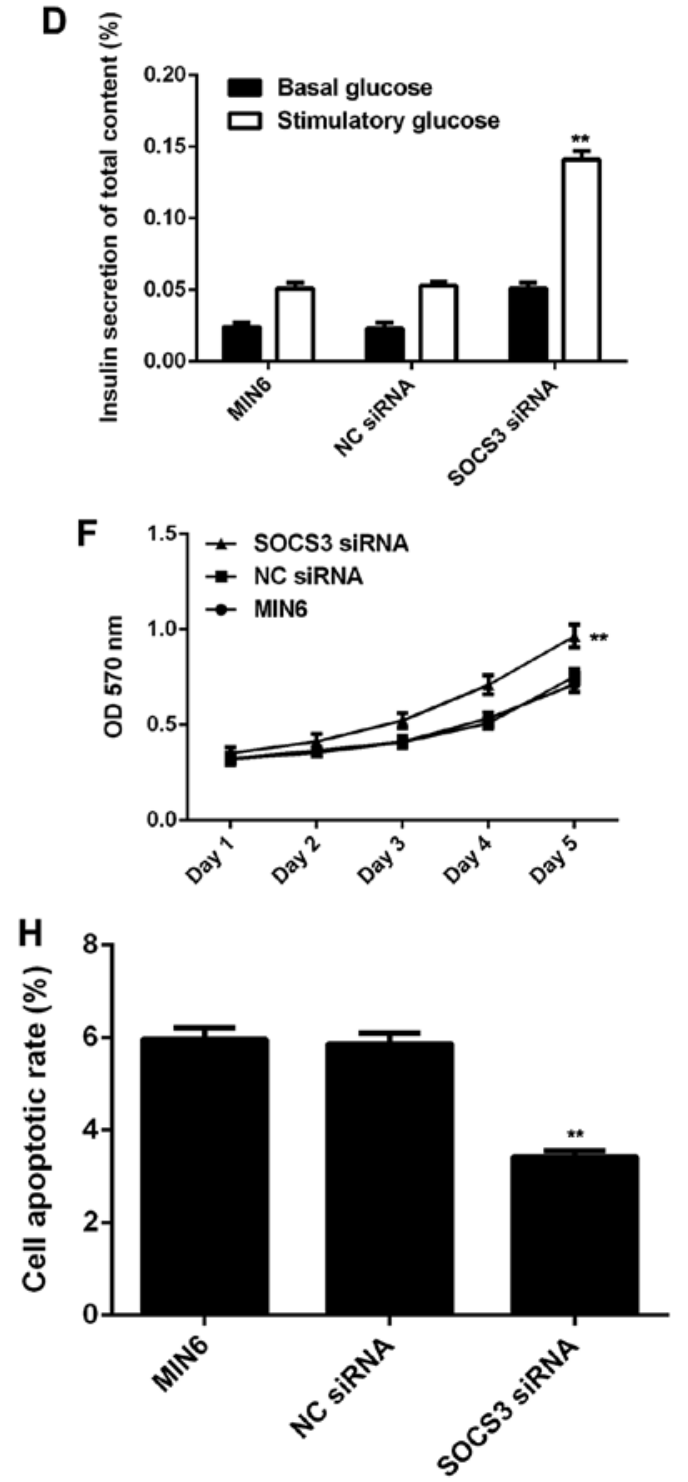

Figure 4. Western blot analysis was used to detect the protein expression of SOCS3 in (A) INS-1 and (B) MIN6 cells transfected with negative control miR mimic (miR-NC) or miR-19a-3p mimic. Untransfected INS-1 or MIN6 cells were used as controls. (C and D) Glucose-stimulated insulin secretion was then determined in each group. (E and F) MTT assay and ( $\mathrm{G}$ and $\mathrm{H}$ ) flow cytometry were then performed to examine cell proliferation and apoptosis. ${ }^{* *} \mathrm{P}<0.01 \mathrm{vs}$. INS-1 or MIN6INS-1 or MIN6 untransfected cells (bars labeled as INS-1 or MIN6) or negative control (NC)-transfected cells. 
A

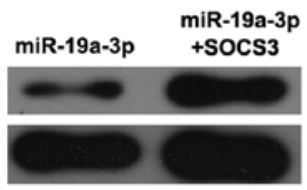

socs 3

INS-1
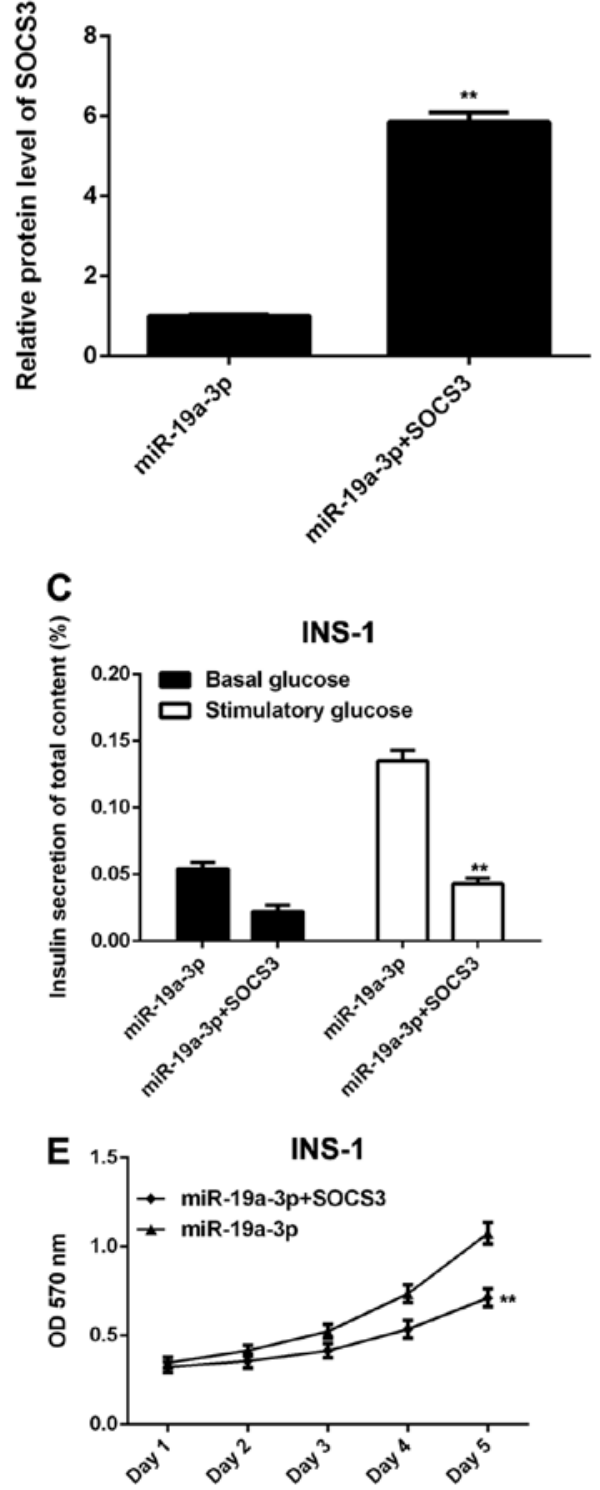

G

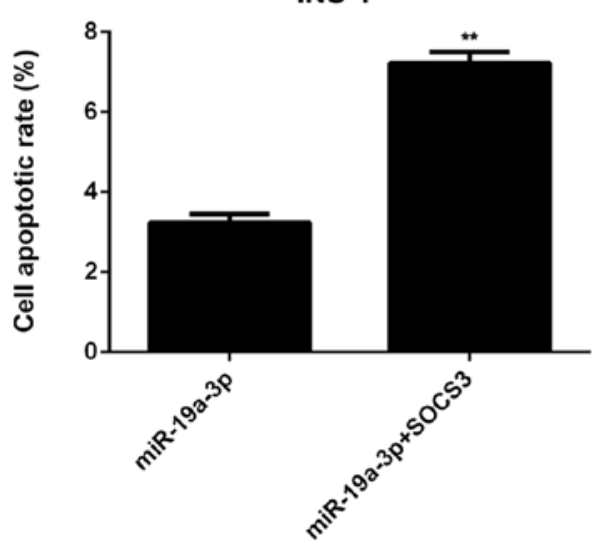

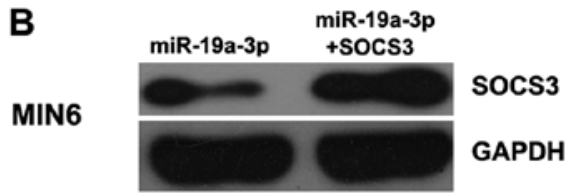
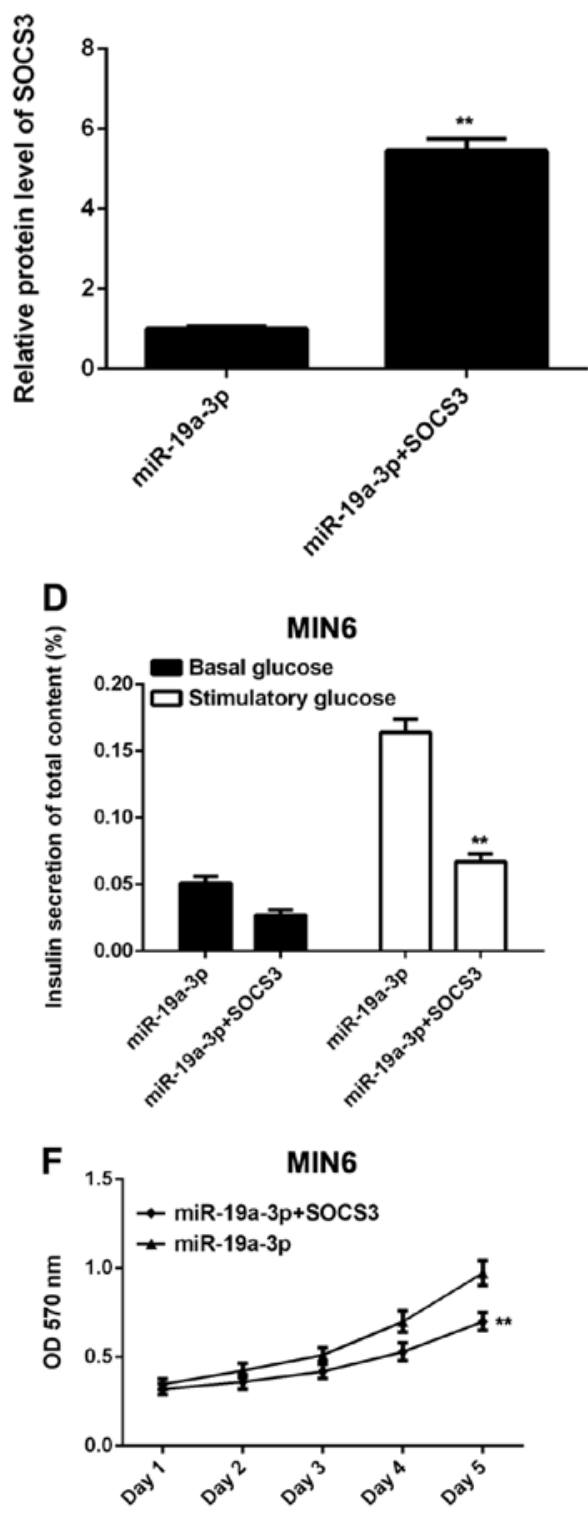

H

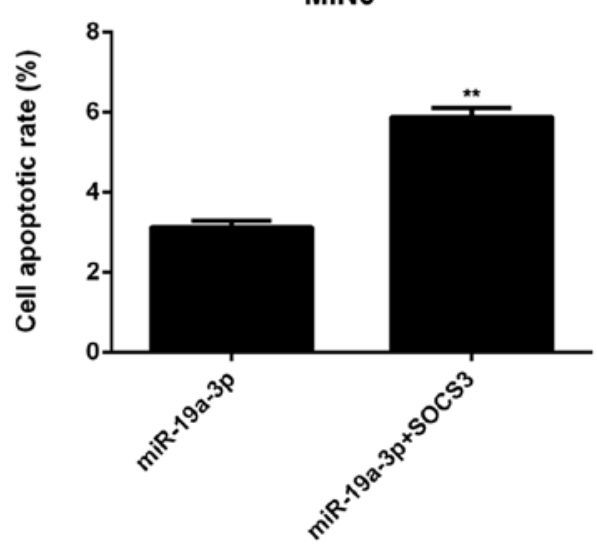

Figure 5. Western blot analysis was used to detect the protein expression of SOCS3 in (A) INS-1 and (B) MIN6 cells transfected with miR-19a-3p mimic, or co-transfected with miR-19a-3p mimic and SOCS3 expression plasmid. (C and D) Glucose-stimulated insulin secretion was then determined in each group. (E and F) MTT assay and $(\mathrm{G}$ and $\mathrm{H})$ flow cytometry were then performed to examine cell proliferation and apoptosis. ${ }^{* *} \mathrm{P}<0.01$ vs. cells transfected with miR-19a-3p and the negative control vector (bars labeled as miR-19a-3p). 

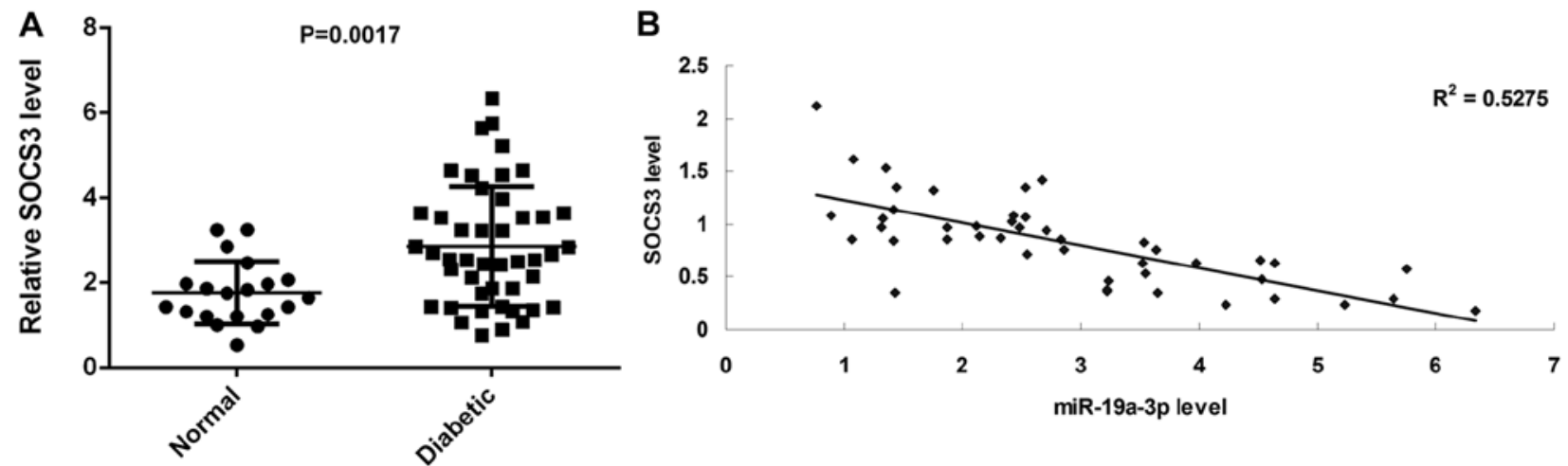

Figure 6. (A) Enzyme-linked immunosorbent assay (ELISA) was used to detect the SOCS3 level in the blood samples from patients with type 2 diabetes (n=45) and normal subjects $(n=20)$. (B) A significant inverse correlation was observed between the plasma miR-19a-3p level and blood glucose concentration among patients with type 2 diabetes $(n=45)$.

colorectal adenocarcinoma (19). In the present study, we found that miR-19a-3p promoted the proliferation and insulin secretion, while it suppresses the apoptosis of pancreatic $\beta$ cells. Jiang et al reported that miR-19a protected endothelial cells from lipopolysaccharide (LPS)-induced apoptosis through the apoptosis signal-regulating kinase 1 (ASK1)/p38 pathway (27). Therefore, the ASK $1 / \mathrm{p} 38$ pathway may also be involved in the protective effects of miR-19a-3p against pancreatic $\beta$ cell apoptosis; this warrants further investigation in future studies.

SOCS3 is a member of the SOCS family, which are negative regulators of cytokine signal transduction and inhibit the cytokine-induced activation of signal transducer and activator of transcription (Stat) signaling (28). SOCS3 has been demonstrated to be associated with the development of leptin resistance and the inhibition of insulin $(29,30)$. The muscle-specific overexpression of SOCS3 in mice has been shown to lead to impaired systemic and muscle-specific glucose homeostasis and insulin function, as well as to decreased basal and leptin-stimulated activity and the phosphorylation of $\alpha 2$ AMP-activated protein kinase ( $\alpha 2 \mathrm{AMPK})$ and acetyl-CoA carboxylase (31). Moreover, muscle SOCS3 overexpression also suppresses leptin-regulated genes involved in fatty acid oxidation and mitochondrial function (31). In the present study, we identified SOCS3 as a direct target gene of miR-19a-3p, and the overexpression of miR-19a-3p led to a significant decrease in the protein level of SOCS3. Moreover, we found that the effects of miR-19a-3p on the proliferation, apoptosis and insulin secretion of pancreatic $\beta$ cells occurred directly through the targeting of SOCS3. it has also previously been reported that SOCS3 is involved in the regulation of pancreatic cell proliferation and apoptosis. The restoration of SOCS3 expression using a demethylating agent (5-aza-2'-deoxycytidine), was shown to markedly suppress the proliferation and to induce the apoptosis of methylated pancreatic cells (32). In addition, we observed a negative correlation between miR-19a-3p expression and the SOCS3 level in the plasma samples of patients with diabetes, which further supports the notion that the protective role miR-19a-3p in diabetes at least partly involves the inhibition of SOCS3 expression.

In conclusion, the findings of the present study demonstrate that miR-19a-3p is significantly downregulated in diabetic patients, and that its expression is inversely correlated with the blood glucose concentration. Moreover, our data indicate that miR-19a-3p significantly enhances the proliferation and insulin secretion, while it inhibits the apoptosis of pancreatic $\beta$ cells (INS-1 and MIN6) by directly targeting SOCS3. Accordingly, miR-19a-3p may become a potential candidate for the treatment of diabetes. However, further studies are warranted to verify our findings.

\section{References}

1. Kaul K, Apostolopoulou M and Roden M: Insulin resistance in type 1 diabetes mellitus. Metabolism 64: 1629-1639, 2015.

2. Chatterjee S and Davies MJ: Current management of diabetes mellitus and future directions in care. Postgrad Med J 91: 612-621, 2015.

3. Keane KN, Cruzat VF, Carlessi R, de Bittencourt PI Jr and Newsholme P: Molecular events linking oxidative stress and inflammation to insulin resistance and $\beta$-cell dysfunction. Oxid Med Cell Longev 2015: 181643, 2015.

4. Berge LI and Riise T: Comorbidity between type 2 diabetes and depression in the adult population: Directions of the association and its possible pathophysiological mechanisms. Int J Endocrinol 2015: 164760, 2015.

5. Ambros V: The functions of animal microRNAs. Nature 431: 350-355, 2004.

6. Bhatia P, Raina S, Chugh J and Sharma S: miRNAs: early prognostic biomarkers for type 2 diabetes mellitus? Biomark Med, 2015.

7. Libânio D, Dinis-Ribeiro M and Pimentel-Nunes P: Helicobacter pylori and microRNAs: Relation with innate immunity and progression of preneoplastic conditions. World J Clin Oncol 6: 111-132, 2015.

8. Bartel DP: MicroRNAs: Genomics, biogenesis, mechanism, and function. Cell 116: 281-297, 2004.

9. Croce CM and Calin GA: miRNAs, cancer, and stem cell division. Cell 122: 6-7, 2005.

10. Martinez-Sanchez A, Nguyen-Tu MS and Rutter GA: DICER inactivation identifies oancreatic $\beta$-cell 'Disallowed' genes targeted by MicroRNAs. Mol Endocrinol 29: 1067-1079, 2015.

11. Latreille M, Herrmanns K, Renwick N, Tuschl T, Malecki MT, McCarthy MI, Owen KR, Rülicke T and Stoffel M: miR-375 gene dosage in pancreatic $\beta$ cells: Implications for regulation of $\beta$ cell mass and biomarker development. J Mol Med Berl 93: 1159-1169, 2015.

12. Jacovetti C, Matkovich SJ, Rodriguez-Trejo A, Guay C and Regazzi R: Postnatal $\beta$ cell maturation is associated with isletspecific microRNA changes induced by nutrient shifts at weaning. Nat Commun 6: 8084, 2015.

13. Al-Kafaji G, Al-Mahroos G, Alsayed NA, Hasan ZA, Nawaz S and Bakhiet M: Peripheral blood microRNA-15a is a potential biomarker for type 2 diabetes mellitus and pre-diabetes. Mol Med Rep 12: 7485-7490, 2015.

14. Busch S, Auth E, Scholl F, Huenecke S, Koehl U, Suess B and Steinhilber D: 5-Lipoxygenase is a direct target of miR-19a-3p and miR-125b-5p. J Immunol 194: 1646-1653, 2015. 
15. Yang J, Zhang Z, Chen C, Liu Y, Si Q, Chuang TH, Li N, Gomez-Cabrero A, Reisfeld RA, Xiang R and Luo Y: MicroRNA19a-3p inhibits breast cancer progression and metastasis by inducing macrophage polarization through downregulated expression of Fra-1 proto-oncogene. Oncogene 33: 3014-3023, 2014.

16. Zhi F, Shao N, Wang R, Deng D, Xue L, Wang Q, Zhang Y, Shi Y, Xia X, Wang S, et al: Identification of 9 serum microRNAs as potential noninvasive biomarkers of human astrocytoma. Neuro Oncol 17: 383-391, 2015.

17. Ibarrola-Villava M, Llorca-Cardeñosa MJ, Tarazona N, Mongort C, Fleitas T, Perez-Fidalgo JA, Roselló S, Navarro S, Ribas G and Cervantes A: Deregulation of ARID1A, CDH1, cMET and PIK3CA and target-related microRNA expression in gastric cancer. Oncotarget 6: 26935-26945, 2015.

18. Balci S, Ayaz L, Gorur A, Yildirim Yaroglu H, Akbayir S, Dogruer Unal N, Bulut B, Tursen U and Tamer L: microRNA profiling for early detection of nonmelanoma skin cancer. Clin Exp Dermatol 41: 346-351, 2016.

19. Zheng G, Du L, Yang X, Zhang X, Wang L, Yang Y, Li J and Wang C: Serum microRNA panel as biomarkers for early diagnosis of colorectal adenocarcinoma. Br J Cancer 111: 1985-1992, 2014

20. Zhu Y, Tian F, Li H, Zhou Y, Lu J and Ge Q: Profiling maternal plasma microRNA expression in early pregnancy to predict gestational diabetes mellitus. Int J Gynaecol Obstet 130: 49-53, 2015.

21. Dou L, Meng X, Sui X, Wang S, Shen T, Huang X, Guo J, Fang W, Man Y, Xi J and Li J: miR-19a regulates PTEN expression to mediate glycogen synthesis in hepatocytes. Sci Rep 5: 11602, 2015.

22. Chakraborty C, George Priya Doss C and Bandyopadhyay S: miRNAs in insulin resistance and diabetes-associated pancreatic cancer: The 'minute and miracle' molecule moving as a monitor in the 'genomic galaxy'. Curr Drug Targets 14: 1110-1117, 2013.

23. Wang X, Wang L, Mo Q, Jia A, Dong Y and Wang G: A positive feedback loop of p53/miR-19/TP53INP1 modulates pancreatic cancer cell proliferation and apoptosis. Oncol Rep 35: 518-523, 2016.
24. Tan Y, Yin H, Zhang H, Fang J, Zheng W, Li D, Li Y, Cao W, Sun C, Liang Y, et al: Sp1-driven up-regulation of miR-19a decreases RHOB and promotes pancreatic cancer. Oncotarget 6: 17391-17403, 2015.

25. Zheng Y, Wang Z, Tu Y, Shen H, Dai Z, Lin J and Zhou Z: miR-101a and miR-30b contribute to inflammatory cytokinemediated $\beta$ cell dysfunction. Lab Invest 95: 1387-1397, 2015.

26. Leung CM, Chen TW, Li SC, Ho MR, Hu LY, Liu WS, Wu TT, Hsu PC, Chang HT and Tsai KW: MicroRNA expression profiles in human breast cancer cells after multifraction and single-dose radiation treatment. Oncol Rep 31: 2147-2156, 2014.

27. Jiang WL, Zhang YF, Xia QQ, Zhu J, Yu X, Fan T and Wang F: MicroRNA-19a regulates lipopolysaccharide-induced endothelial cell apoptosis through modulation of apoptosis signal-regulating kinase 1 expression. BMC Mol Biol 16: 11, 2015.

28. Kim MH, Kim MS, Kim W, Kang MA, Cacalano NA, Kang SB, Shin YJ and Jeong JH: Suppressor of cytokine signaling (SOCS) genes are silenced by DNA hypermethylation and histone deacetylation and regulate response to radiotherapy in cervical cancer cells. PLoS One 10: e0123133, 2015.

29. Wunderlich CM, Hövelmeyer N and Wunderlich FT: Mechanisms of chronic JAK-STAT3-SOCS3 signaling in obesity. JAKSTAT 2: e23878, 2013

30. Krebs DL and Hilton DJ: A new role for SOCS in insulin action. Suppressor of cytokine signaling. Sci STKE 2003: PE6, 2003.

31. Yang Z, Hulver M, McMillan RP, Cai L, Kershaw EE, Yu L, Xue B and Shi H: Regulation of insulin and leptin signaling by muscle suppressor of cytokine signaling 3 (SOCS3). PLoS One 7: e47493, 2012

32. Wang J, Zhou H, Han Y, Liu X, Wang M, Wang X, Yin G, Li X and Xiang M: SOCS3 methylation in synergy with Reg3A overexpression promotes cell growth in pancreatic cancer. J Mol Med Berl 92: 1257-1269, 2014. 